\title{
Mathematical model of tumour cord growth along the source of nutrient
}

\author{
S. Astanin ${ }^{1}$, A. Tosin \\ Dipartimento di Matematica, Politecnico di Torino, \\ Corso Duca degli Abruzzi 24, Turin, 10129, Italy
}

\begin{abstract}
A mathematical model of the tumour growth along a blood vessel is proposed. The model employs the mixture theory approach to describe a tissue which consists of cells, extracellular matrix and liquid. The growing tumour tissue is supposed to be surrounded by the host tissue. Tumours where complete oxydation of glucose prevails are considered. Special attention is paid to consistent description of oxygen consumption and growth processes based on the energy balance. A finite difference numerical method is proposed. The level set method is used to track an interface between the tissues. The simulations show localization of the tumour within a limited distance from the vessels and constant expansion velocity along the vessels.
\end{abstract}

Key words: tumour growth, hypoxia, tumour cords, tumour-host interface, mathematical model, mixture theory, finite difference, level set method

AMS subject classification: 35R35, 92C10, 92B05, 92C17

\section{Introduction}

Cancer disease manifests itself as abnormally excessive cell proliferation. This is the result of dysregulation of normal constraints on cellular proliferation [2].

This fact has serious implications on the morphology of the growth. While most of the cells in the human body are within few cell diameters from a blood vessel, intensive proliferation of tumour cells creates cell populations distant from blood vessels and deprived from nutrient and oxygen supply [22]. This leads to formation of cylindrical structures around blood vessels. These structures are composed of tumour cells and are often surrounded by regions of necrosis. These

\footnotetext{
${ }^{1}$ Corresponding author. E-mail: astanin@calvino.polito.it
} 
structures are referred to as tumour cords [32, 20, 23].

Tumour cords are known to have stationary radial structure with the most intensive proliferation near the blood vessel. This proliferation pushes tumour cells further away from the vessel, until they reach a hypoxic or nutrient poor zone, where cells eventually die. The processes of growth and death tend to be in equilibrium as the cord reaches a certain radius. This radial structure has been described in a series of mathematical models [9, 8, 6, 7].

In this model we focus at describing propagation of the cord along the vessel together with formation of its radial structure. Thus the model is essentially multidimensional.

The basic version of the model has already been introduced in [4], and discussed in more detail in [34] based on the theory of deformable porous media. Tumour mass was described as a saturated mixture of proliferating cells, extracellular fluid, and rigid non-remodelling matrix. The latter work proposed quantitative and qualitative analysis of the free boundary problem, which allows to estimate the steady size of the tumour cord in terms of model parameters.

Both of the models above relied on a phenomenological description of the tumour growth and oxygen consumption. In this paper we further extend the model proposing that proliferation of cells should strongly depend on the amount of energy available to the cells, which in turn is regulated by the supply of nutrients. This way we propose a model where growth and oxygen uptake are no longer mere arbitrarily chosen model parameters, but are indeed two strongly coupled processes.

We consider tumour cord as the growth of tumour tissue around the blood vessel and assume that it is surrounded by the normal tissue of the host. We consider these two tissues to be immiscible but exhibiting similar mechanical properties.

Tissues are described as porous media composed of cells, intercellular liquid, and rigid extracellular matrix (ECM) [3]. This approach has been already successfully applied to tumour tissue modelling by [10], who deal with a biphasic mixture and neglect ECM, and [11], who deal with triphasic mixture. Both tissues in our model may be compressed or stretched and constitute continuous medium.

We neglect differences in the ECM structure between tumour and host. The former is likely to produce looser ECM, and thus demonstrate different mechanical properties than the latter.

We assume that growth and death occur only in tumour tissue, and thus suppose that tumour is a major consumer of nutrients and oxygen.

There is flow of nutrient and oxygen from the vessel through the tissue. While normal regulation of cell proliferation in tumour may be broken, its growth is still certainly limited by availability of nutrients and oxygen, and by maximum achievable cell packing density. The death is result of insufficient supply of nutrients and oxygen. The growth is likely to be limited by either supply of oxygen or supply of one of the nutrients. For the sake of definiteness we shall assume oxygenlimited growth, but the model would be practically the same for a highly diffusive nutrient.

Our approach allows to describe tumour cord propagation along the vessel as well as its morphology, formation of hypoxic regions and implications of growth on the tissue density and nutrient distribution.

Tumour growth is a very complex process, and our model grasps only the minimal range of phenomena required to describe such form of an anisotropic growth as tumour cords (Figure 1).

The model is limited to solely macroscopic phenomenological description of tissue properties 


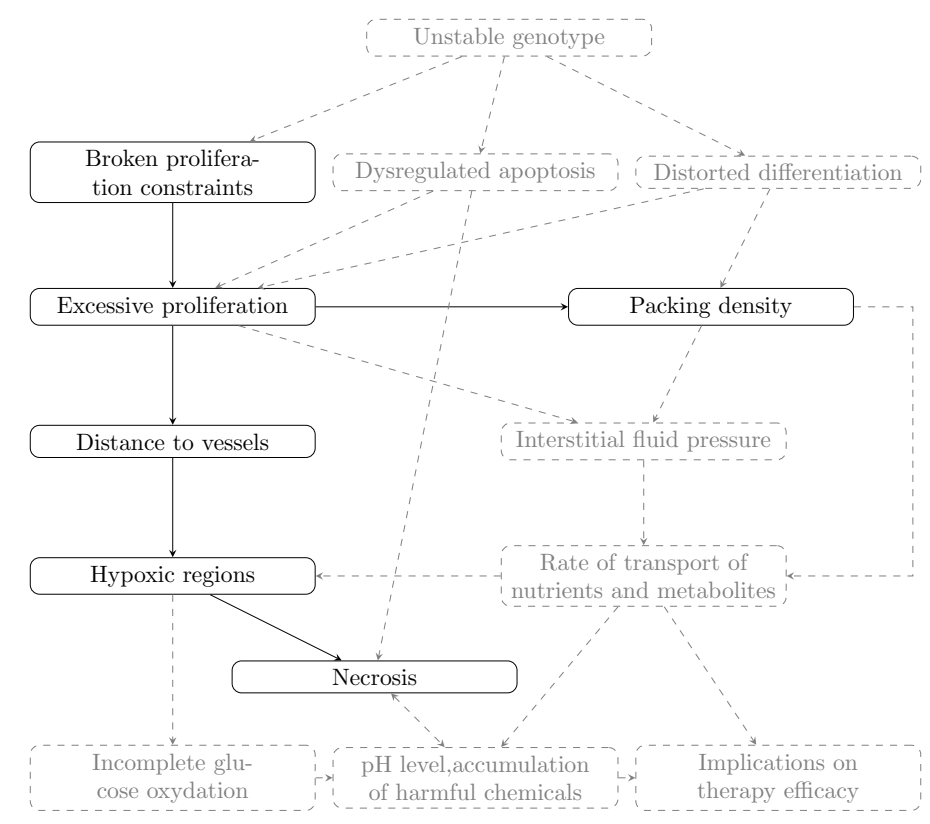

Figure 1: Scope of the model. Dashed boxes outline some phenomena and relations which are probably important for tumour cord development and tumour therapy but are not covered by the model.

and growth. Related effects like displacement of blood vessels, destruction of the surrounding normal tissue, and formation and deformation of the ECM with abnormal properties, are neglected in the model presented here.

According to [2], four cell functions tend to be incorrectly regulated in neoplasm: normal constraints on cell proliferation, differentiation programme, stability of genetic organization, and apoptosis. As far as only excessive proliferation of tumour tissue is concerned, the model takes into account only inefficacy of proliferation constraints. This leaves out of scope of the model such phenomena as evolution of tumour behaviour with time (mutable genotype) and changes of tissue properties due to incomplete differentiation programme.

However even this simple approach allows to explain appearance of hypoxic or nutrientdeprived regions and changes of packing density inside the tumour. Within the framework of the model these effects drive growth of the tumour cord in anisotropic mode.

\section{Tissue model}

Figure 2 represents the cord schematically. For modelling purpose axial symmetry of the cord may often be assumed.

We assume that the tissue may be described as a saturated porous medium consisting of three phases: cells, extracellular liquid, and rigid extracellular matrix. 


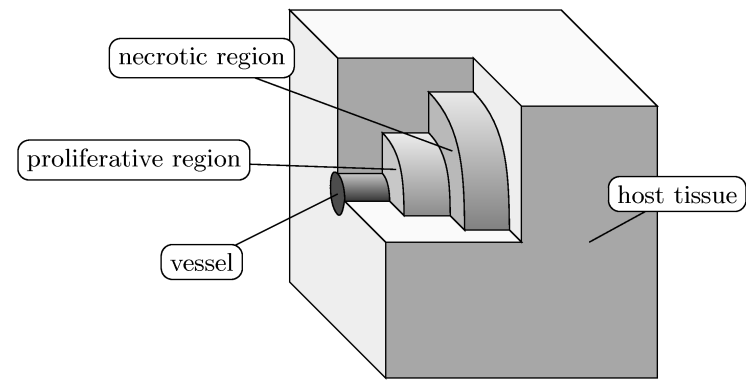

(a)

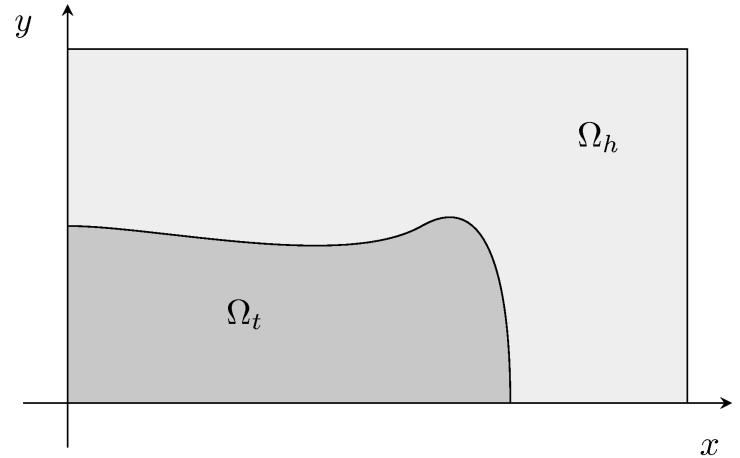

(b)

Figure 2: (a) Cord scheme. Proliferative region surrounds the vessel, necrotic region may form outside. Cells migrate from inside outside. The cord is surrounded by host tissue. (b) Tumour cord region $\Omega_{t}$ and region of host tissue $\Omega_{h}$. Blood vessel is positioned along the $x$-axis.

Let us consider a tumour located in a finite region of the plane. We describe tumour geometry with a two-dimensional connected domain $\Omega_{t}(t)$ in the rectangle $\Omega=\left\{x, y \mid 0 \leq x \leq x_{\max }, 0 \leq y \leq y_{\max }\right\}$. We assume that there are blood vessels which coincide with some of the boundaries. In particular we shall consider the case when there is a vessel along $x$-axis (Figure 2). This configuration is different from that described in [6, 7], where ensembles of parallel identical cords are considered, and the host tissue is not present between them. We instead consider a single cord surrounded by the normal tissue.

Tumour domain $\Omega_{t}$ evolves with time $t$. Its complement domain $\Omega_{h}(t)=\Omega \backslash \Omega_{t}(t)$ is considered to be filled with host tissue. We define mechanical properties of both domains in the same way.

The following variables describe the tissue: $\phi=\phi(\boldsymbol{x}, t)$ - volume fraction of cells, $\boldsymbol{v}=$ $\boldsymbol{v}(\boldsymbol{x}, t)$ - velocity field of the cell phase, $\phi_{l}=\phi_{l}(\boldsymbol{x}, t)$ - volume fraction of extracellular liquid, $\boldsymbol{v}_{l}=\boldsymbol{v}_{l}(\boldsymbol{x}, t)$ - velocity field of the liquid phase, $\phi_{E C M}$ - fixed volume fraction of rigid extracellular matrix. We denote by $\boldsymbol{x}$ a point on the plane $\boldsymbol{x}=(x, y) \in \Omega$.

We enforce the mixture saturation condition

$$
\phi+\phi_{l}+\phi_{E C M}=1
$$

for all $\boldsymbol{x} \in \Omega$. Given that $\phi_{E C M}=$ const in time (no matrix degradation/deposition), one may write also

$$
\phi+\phi_{l}=\phi_{*}=\text { const. }
$$

We have to point out that $\phi$ in the tumour domain $\Omega_{t}$ is the volume fraction of tumour cells, while $\phi$ in the host tissue domain $\Omega_{h}$ is the volume fraction of host tissue cells. As we shall see, tissues remain segregated, hence a single state variable is sufficient to track the evolution of the cell population in either domain. The next two sections discuss mechanical issues common to both domains. The subsequent sections discuss questions specific to tumour tissue and tumour-host interface. 


\section{Mass and stress balance}

Following [1] we use the following mass balance equations, assuming non-compressibility of liquid and cellular phase and the same density $\rho$ for all components:

$$
\begin{aligned}
\frac{\partial \phi}{\partial t}+\operatorname{div}(\phi \boldsymbol{v}) & =\frac{\Gamma}{\rho}, \\
\frac{\partial \phi_{l}}{\partial t}+\operatorname{div}\left(\phi_{l} \boldsymbol{v}_{l}\right) & =\frac{\Gamma_{l}}{\rho},
\end{aligned}
$$

where $\Gamma, \Gamma_{l}$ are growth rates for cells and liquid phases respectively. As $\rho=$ const, henceforth we shall refer to volume ratios $\phi$ and $\phi_{l}$ as cell packing densities or just density, though we should keep in mind that the physical density of the cells and the liquid is $\rho$.

One may also add the third component representing the mass of dead cells, not yet converted into the extracellular liquid. We assume that this conversion is much faster than growth and death of the cells, and the mass of not yet dissolved dead cells is negligible.

In other words, we assume that dead cells are completely and immediately dissolved in the extracellular liquid, and that extracellular liquid is captured upon cellular growth and proliferation $\left(\Gamma=-\Gamma_{l}\right)$. This allows to sum (2.2) and (2.3) and obtain the following mass balance equation for the whole mixture:

$$
\operatorname{div}\left(\phi \boldsymbol{v}+\phi_{l} \boldsymbol{v}_{l}\right)=0 .
$$

As cell growth and movement are much slower than the mechanical stress relaxation, we neglect inertia and use quasi-static approximation for mechanical stress balance:

$$
\begin{aligned}
-\operatorname{div}(\phi \mathbb{T}) & =\boldsymbol{m}^{\sigma}, \\
-\operatorname{div}\left(\phi_{l} \mathbb{T}_{l}\right) & =\boldsymbol{m}_{l}^{\sigma} .
\end{aligned}
$$

In these equations $\mathbb{T}$ and $\mathbb{T}_{l}$ are Cauchy stress tensors for cellular and liquid phases respectively, and $\boldsymbol{m}^{\sigma}$ and $\boldsymbol{m}_{l}^{\sigma}$ are interaction forces exerted on the constituents by the other constituents of the mixture.

We suppose that the tissue is isotropic and use symmetrical stress tensors

$$
\begin{aligned}
\mathbb{T} & =-(p+\Sigma(\phi)) \mathbb{I}, \\
\mathbb{T}_{l} & =-p \mathbb{I},
\end{aligned}
$$

where $p=p(\boldsymbol{x}, t)$ is the interstitial liquid pressure, and the function $\Sigma(\phi)$ describes intercellular interactions, being positive for compressed tissue.

Depending on the cell type one may observe stresses not only when the tissue is compressed, but also when it is stretched. These stresses depend on intercellular binding forces. We assume that $\Sigma(\phi)$ is monotonic and changes sign in $\left[0 ; \phi_{*}\right]$. The point $\phi_{0}$ where $\Sigma\left(\phi_{0}\right)=0$ is the density of relaxed state. We may also consider a continuous function $\Sigma$, such that $\Sigma(\phi) \equiv 0$, for $\phi \leq \phi_{0}$. This function would describe a non-connective tissue with no binding forces between the separated cells. $\Sigma(\phi)$ may also be chosen to be vanishing for $\phi$ below a certain threshold, to describe a 
situation when the cells are so scarce, than no longer interact. Selection of $\Sigma$ was discussed in more details in [19].

According to general principles of the theory of mixtures, the interaction forces between the constituents may be described as

$$
\begin{aligned}
& \boldsymbol{m}^{\sigma}=p \nabla \phi-\Lambda \boldsymbol{v}+\Lambda_{l}\left(\boldsymbol{v}_{l}-\boldsymbol{v}\right) \\
& \boldsymbol{m}_{l}^{\sigma}=p \nabla \phi_{l}-\Lambda_{l}\left(\boldsymbol{v}_{l}-\boldsymbol{v}\right)
\end{aligned}
$$

Viscous friction force $-\Lambda \boldsymbol{v}$ describes adhesion of the cells to the matrix, where $\boldsymbol{v}$ is their relative velocity. We neglect friction between the liquid phase and the matrix, assuming it to be much smaller than cell-matrix friction. Cell-liquid interaction is described by the term $\pm \Lambda_{l}\left(\boldsymbol{v}_{l}-\boldsymbol{v}\right)$.

Assuming that $\Lambda_{l}=\phi_{l}^{2} / \hat{k}$, where $\hat{k}$ is the medium permeability, (2.6), (2.8) and (2.10) give us a relation between the velocity of the liquid phase and that of the cells:

$$
\boldsymbol{v}_{l}=\boldsymbol{v}-\frac{\hat{k}}{\phi_{l}} \nabla p
$$

which expresses Darcy's law.

In the same way equations (2.5), (2.7), (2.9) and (2.11), for $\phi_{l}=\phi_{*}-\phi$, imply

$$
\boldsymbol{v}=-\frac{1}{\Lambda}\left(\phi_{*} \nabla p+\nabla(\phi \Sigma(\phi))\right) .
$$

Substituting these expressions for velocities of the compounds in (2.4), we obtain an equation for $\phi$ and $p$ :

$$
\operatorname{div}\left(\left(\frac{\phi_{*}^{2}}{\Lambda}+\hat{k}\right) \nabla p+\frac{\phi_{*}}{\Lambda} \nabla(\phi \Sigma(\phi))\right)=0 .
$$

Substituting $\boldsymbol{v}$ into (2.2), we obtain an additional equation for the same variables $\phi$ and $p$ :

$$
\frac{\partial \phi}{\partial t}-\operatorname{div}\left(\frac{\phi}{\Lambda}\left(\phi_{*} \nabla p+\nabla(\phi \Sigma(\phi))\right)\right)=\frac{\Gamma}{\rho} .
$$

The model (2.12-2.13) may be simplified in an assumption that friction between cells and liquid is relatively small. This means that the terms $\pm \Lambda_{l}\left(\boldsymbol{v}_{l}-\boldsymbol{v}\right)$ in equations (2.9-2.10) are negligible. As a consequence, $\nabla p$ is the same order of magnitude, hence negligible. Thus equation (2.13) is reduced to only one equation for the variable $\phi$ :

$$
\frac{\partial \phi}{\partial t}-\operatorname{div}\left(\frac{\phi}{\Lambda} \nabla(\phi \Sigma(\phi))\right)=\frac{\Gamma}{\rho} .
$$

Equation (2.12) may then be solved after solving (2.14) to evaluate the effects of increased interstitial fluid pressure (IFP) inside the tumour, which may have implications on the rate of chemicals transport and removal from the tumour extracellular space [26]. However these phenomena are out of scope of the model presented here. 
One may notice, that the depending on the choice of $\Sigma$ equation (2.14) may become an inverse diffusion equation for some $\phi$. This may be avoided if we ensure that the solution stays in the range where

$$
\phi \Sigma(\phi)+\phi^{2} \Sigma^{\prime} \geq 0
$$

However the choice of $\Sigma$ is determined by phenomenological considerations, and the model stays valid only for those values of $\phi$ for which those considerations make sense. These restrictions may in fact be even stricter than those imposed by mathematical properties of the model.

For example, if we chose $\Sigma=\phi-\phi_{0}$, we expect that that the solution will stay close to the zero stress value $\phi_{0}$, and linear elasticity approximation is still valid. The mathematical problem above is not well posed for $\phi \in\left(0, \phi_{0} / 2\right)$, but from the physiological point of view even $\phi=\phi_{0} / 2$ is highly abnormal, as it represents the tissue stretched two times. Describing such a tissue will likely require choosing a different $\Sigma$ at least or using an absolutely different approach.

Thus the range of solution for which the model is adequate may be even narrower than the range where the related mathematical problem is well posed. In fact the model is used only for simulations where $\phi \approx \phi_{0}$.

\section{Energy balance}

A biological tissue is essentially an open system. It depends on the continuous inflow of the nutrients to the tissue, which serves both as building materials for the tissue and as a source of energy.

Even simple maintenance of life functions requires energy. Given that the key feature of tumour tissue is growth, we should also consider the energy required for cell duplication.

We assume that most of the energy is supplied to the tissue in the form of glucose. This is not exactly true, as there are other energy sources like amino acids and lipids. However it is D-glucose that plays a central role in metabolism.

The net energy gain of glucose may vary according to local conditions. Full oxidation would produce up to $2840 \mathrm{~kJ}$ per mole of glucose but requires 6 molecules of oxygen per molecule of glucose. This is approximately equivalent to 30-32 molecules of ATP per molecule of glucose [27].

When there is lack of oxygen only the first part of the catabolic pathway is feasible. Glycolysis produces only two molecules of ATP per molecule of glucose but does not require oxygen. Pyruvate produced during glycolysis is further transformed into lactic acid in this case (Figure 3).

This could be expressed as two concurrent reactions (actually, the chain of reactions is much longer and less straightforward). The full oxidation might be expressed as

$$
\mathrm{Glc}+6 \mathrm{O}_{2} \longrightarrow 6 \mathrm{CO}_{2}+N \mathrm{ATP},
$$

where $N \approx 30-32$ is the number of ATP molecules produced during complete oxydation of glucose. In [31] $N=36$ is suggested.

The partial (anaerobic) oxidation of glucose is then expressed as

Glc $\longrightarrow 2$ Lactic acid +2 ATP. 


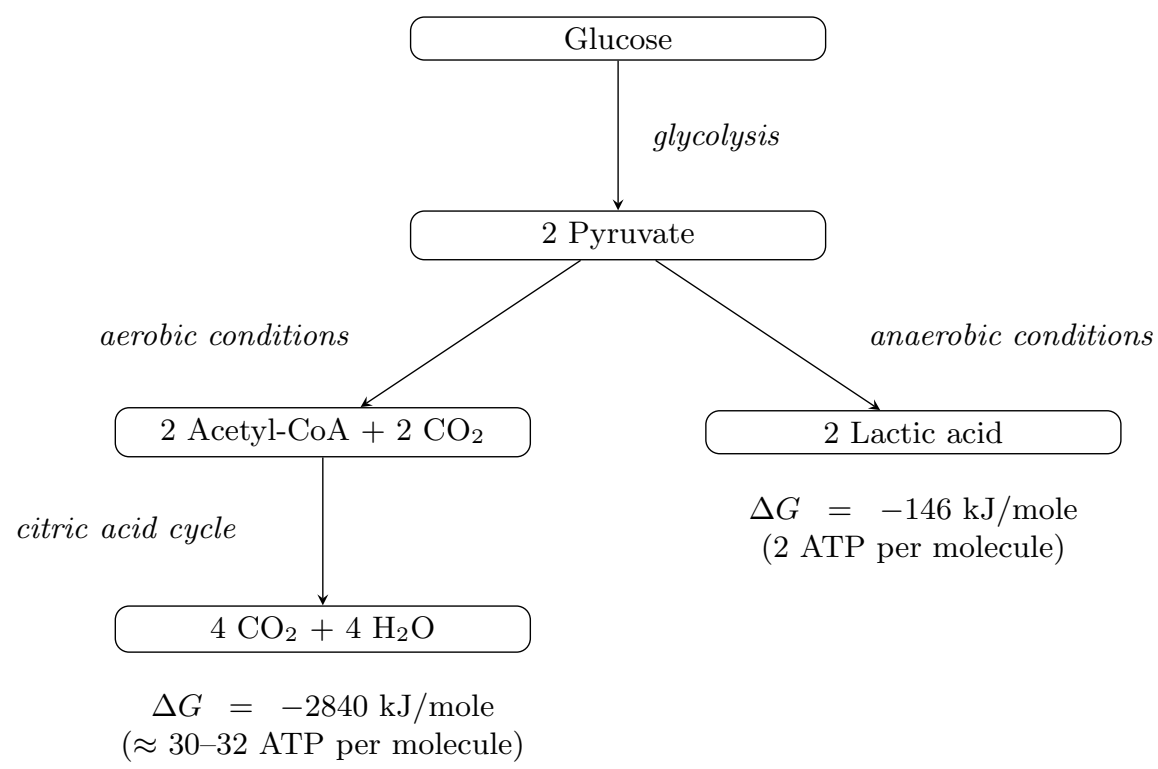

Figure 3: Glucose catabolic pathways (based on [27]). Anaerobic pathway is less energy efficient and in addition it acidifies the microenvironement.

While anaerobic pathway is less energy efficient (the yield is about $5 \%$ of that in the case of full oxidation), it proved to give an evolutionary advantage to tumours, as they tend to develop and heavily rely on the ability for glycolysis oriented metabolism [31, 18, 17].

In our model we assume that only the complete oxydation takes place, and the tumour is yet incapable of altered glucose catabolism. This practically means that the rate of ATP (energy carrier molecule) production is proportional to the rate of oxygen consumption:

$$
N \frac{d[\mathrm{ATP}]}{d t}=-6 \frac{d\left[\mathrm{O}_{2}\right]}{d t}
$$

This observation allows to consider in the model only oxygen concentration because it effectively determines the amount of energy available for the cells.

On a microscopic (cellular) scale one might observe accumulation of energy in the form of both glycogen reserve and ATP accumulation at G1 and G2 phases of the cell cycle. We assume that on a macroscopic (tissue) scale these effects of accumulation of energy carriers within individual cells are less important. In other words the net energy production averaged over the whole cell cycle and a large number of cells is zero.

This assumption means that at a macroscopic scale all the energy produced is immediately used. Within this model we assume that there are two uses of the energy:

- maintenance of minimal life functions,

- cellular division and growth. 
From the microscopic point of view the former is more or less the cost of staying in G0 phase, while the latter is the cost of completing the full cell cycle. We shall address energy consumption issues in more detail in the section on proliferation, death and oxygen consumption.

\section{Distribution of oxygen}

In those tumours where complete oxydation of glucose prevails the variable of interest is the amount of oxygen. Let $c(\boldsymbol{x}, t)$ be the concentration of oxygen.

Oxygen is one of few biological chemicals which distribution in the tissue is governed by simple Fick's law of diffusion, as it may freely diffuse through cellular membranes [27].

Although the diffusion rate of oxygen through cellular membranes may be lower than in the free liquid, we assume that the concentration of oxygen inside the cells is the same as in the extracellular liquid.

Oxygen diffuses in the tissue and is consumed by tumour cells with a rate $q(\boldsymbol{x}, c, \phi)$ :

$$
\frac{\partial c}{\partial t}=D \Delta c+\mathrm{q}(\boldsymbol{x}, c, \phi)
$$

where $D$ is the diffusion coefficient of the oxygen.

We expect oxygen consumption in quiescent and actively proliferating cells to be different. As $\mathrm{q}(\boldsymbol{x}, c, \phi)$ is likely to depend on the rate of cell proliferation, we postpone its discussion until the section about cell proliferation.

We consider oxygen diffusion to be a much faster process than tumour growth. Thus the distribution of oxygen in the tissue is quasistatic. For this reason we neglect time derivative and use Poisson equation to describe oxygen distribution for any given tumour configuration:

$$
D \Delta c=-\mathrm{q}(\boldsymbol{x}, c, \phi) .
$$

The sources of oxygen are blood vessels. We assume that the concentration of oxygen inside the vessels is constant. From the modelling point of view this is equivalent to applying Dirichlet boundary conditions for the concentration of oxygen on the surface of the vessels (boundaries of the domain).

\section{Proliferation, death, and oxygen consumption}

As we noted, excessive cell proliferation is the key feature of tumour development. Proliferation and cell death under the conditions of non-uniform nutrient distribution play a crucial role in the formation of the stationary structure of the tumour cord $[6,7]$.

Thus it is essential to reflect the interdependency of the growth/death processes and the quantity of oxygen and nutrients available. However it is still an open question how proliferation and death processes depend on nutrient distribution.

We propose that a key to understand these processes is energy balance.

Let $\dot{Q}_{A}(\boldsymbol{x}, t)$ be the rate of ATP production:

$$
\dot{Q}_{A}=\kappa \phi g(c)
$$


where $\kappa$ is the integral reaction rate of glucose oxidation, and $g(c)$ defines the effectiveness of the oxidative processes as a function of oxygen supply.

One might expect that

$$
\mathrm{g}(c) \approx \frac{c}{c_{x}}, \quad \text { for } c \ll c_{x} .
$$

where $c_{x}$ is a threshold oxygen concentration above which the production of ATP is limited by physiological constraints of the cells rather than by supply of oxygen. An example of such a function would be

$$
g(c)=\tanh \left(\frac{c}{c_{x}}\right)
$$

or the classical Michaelis-Menten rule

$$
\mathrm{g}(c)=\frac{c}{c_{x}+c}
$$

Let $\dot{Q}_{0}$ be the rate of ATP consumption due to the life maintenance needs. If there is no accumulation of ATP (see Energy balance section), the concentration of ATP is constant, and the total rate of ATP consumption depends only on the amount of cells consuming:

$$
\dot{Q}_{0}=-\hat{\theta} \phi
$$

where $\hat{\theta}$ is a total rate of ATP consumption per cell under the given conditions.

Then the rate of ATP uptake for the purpose of growth and division would be

$$
-\dot{Q}_{M}=\dot{Q}_{A}+\dot{Q}_{0}=(\kappa \mathrm{g}(c)-\hat{\theta}) \phi
$$

This defines the rate of growth as

$$
\Gamma^{+}=\frac{\dot{Q}_{M}}{Q_{M}^{0}} \ln 2=\frac{\ln 2}{Q_{M}^{0}} \phi(\kappa \mathrm{g}(c)-\hat{\theta})_{+},
$$

where $Q_{M}^{0}$ is an average cost of the full cell cycle (duplication, G1 growth, DNA synthesis and G2 growth), and $(\cdot)_{+}$denotes the positive part of $(\cdot)$ :

$$
(\cdot)_{+}=\frac{|\cdot|+(\cdot)}{2} .
$$

Meanwhile the uptake of oxygen is

$$
\mathrm{q}(c, \phi)=-\frac{6}{N} \dot{Q}_{A}=-\frac{6 \kappa}{N} \phi \mathrm{g}(c) .
$$

One of the additional properties of this model is an existence of a critical oxygen level below which proliferation is impossible. Assuming that $\mathrm{g}(c)$ is increasing monotonic function for $c \geq 0$, $\mathrm{g}(0)=0$, let us consider the case when all the ATP produced is used for maintenance of life functions:

$$
\dot{Q}_{A}+\dot{Q}_{0}=\kappa \mathrm{g}(c)-\hat{\theta}=0
$$


If $\kappa>0, \hat{\theta}>0$ then there exists $c_{0}>0$ such that there is no growth for $c \leq c_{0}$ :

$$
\mathrm{g}\left(c_{0}\right)=\frac{\hat{\theta}}{\kappa} .
$$

When $c<c_{0}$, production of ATP is insufficient. We suppose that when production of ATP is insufficient only as many cells survive as may be fed with a given rate of ATP production:

$$
\frac{\phi_{\text {surviving }}}{\phi}=\left|\frac{\dot{Q}_{A}}{\dot{Q}_{0}}\right|=\frac{\kappa g(c)}{\hat{\theta}}, \text { for } c<c_{0}
$$

so that the fraction of dying cells is

$$
\frac{\phi_{\text {dying }}}{\phi}=\left(1-\frac{\kappa \mathrm{g}(c)}{\hat{\theta}}\right)_{+}
$$

This allows to define the death rate as

$$
\Gamma^{-}=-\phi \frac{\ln 2}{\hat{\theta} \tau_{1 / 2}}(\hat{\theta}-\kappa \mathrm{g}(c))_{+}=-\phi \frac{\ln 2}{\hat{\theta} \tau_{1 / 2}}(\kappa \mathrm{g}(c)-\hat{\theta})_{-} \leq 0,
$$

where $\tau_{1 / 2}$ is the "half life" of the dying cells, and $(x)_{-}$is the negative part of $(x):(x)_{-}=(-x)_{+}$.

Finally, we have the following growth/death term for $\phi$ :

$$
\Gamma=\Gamma^{+}+\Gamma^{-}=\phi \frac{\ln 2}{Q_{M}^{0}}(\kappa \mathrm{g}(c)-\hat{\theta})_{+}-\phi \frac{\ln 2}{\hat{\theta} \tau_{1 / 2}}(\kappa \mathrm{g}(c)-\hat{\theta})_{-}
$$

and the following uptake term for $c$ :

$$
\mathrm{q}(c, \phi)=-\frac{6}{N} \kappa \phi \mathrm{g}(c) .
$$

This approach provides a sensible link between oxygen consumption and growth. However, if there is abundant oxygen supply $(\mathrm{g}(c)=1)$ this growth term does not impose any limit on proliferation of cells, yet $\phi$ should never exceed $\phi_{*}$ for it is constrained by (2.1).

Thus a growth term like this can be used in the model only if it is accompanied by a limit on the cellular packing density. For example, this might be achieved using a function $\Sigma(\phi)$ such that $\lim _{\phi \rightarrow \phi_{*}} \Sigma(\phi)=+\infty$. Actually this would describe a situation when cells proliferate no matter how dense the tissue is.

In this paper we do not impose such a requirement. Instead we modify $\dot{Q}_{A}$ to inhibit proliferation (ATP production) in overly dense tissue:

$$
\dot{Q}_{A}=\kappa \phi \mathrm{f}(\phi) \mathrm{g}(c)
$$

where $\mathrm{f}(\phi)$, the inhibition function, vanishes when $\phi=\phi_{*}$. Possible candidates for $\mathrm{f}(\phi)$ are $\mathrm{f}(\phi)=$ $\left(\phi_{*}-\phi\right)$ (this would give logistic growth term for $\mathrm{g}(c) \equiv 1$ ) or $\mathrm{f}(\phi)=H\left(\phi_{*}-\phi\right)$. 
Still maintaining $\dot{Q}_{A}+\dot{Q}_{0}+\dot{Q}_{M}=0$, we have the following net growth term

$$
\Gamma=\Gamma^{+}+\Gamma^{-}=\gamma \phi(\mathrm{f}(\phi) \mathrm{g}(c)-\theta)_{+}-\varepsilon \phi(\theta-\mathrm{f}(\phi) \mathrm{g}(c))_{+},
$$

where $\theta=\hat{\theta} / \kappa$ is the basic upkeep cost per cell, $\gamma=\kappa \ln 2 / Q_{M}^{0}$ is the growth rate, and $\varepsilon=$ $\kappa \ln 2 / \hat{\theta} \tau_{1 / 2}$ is the death rate.

Concerning the oxygen consumption term we have

$$
\mathrm{q}(\phi, c)=-\alpha \phi \mathrm{f}(\phi) \mathrm{g}(c)
$$

where $\alpha=6 \kappa / N$ is oxygen uptake rate.

In this case we do not introduce a critical nutrient value $c_{0}$ any more, because it would depend on the local packing density $\phi$ given that $\dot{Q}_{A}$ is not a linear function of $\phi$.

It is possible to use $\mathrm{f}(\phi)=$ const and $\mathrm{g}(c)=c / c_{x}$, but in this case one should keep in mind that this kind of model is valid only as long as solution $\phi$ stays in the range $\phi \in\left[0, \phi_{*}\right]$.

We assume that there is intensive proliferation in the tumour tissue which is limited only by availability of energy (oxygen and nutrients) and space (reasonable packing density constraints apply also to tumour tissue). We also assume that neither gross proliferation nor cell death takes place in the host tissue. This assumption is probably not very restrictive as most cells in the mature human body are duly differentiated and lost their division ability.

If degradation of the host tissue has to be taken into account, then the degradation term would be essentially the same as for tumour tissue (only as many cells survive as have minimal energy supply):

$$
\Gamma^{-}=-\varepsilon \phi(\theta-\mathrm{f}(\phi) \mathrm{g}(c))_{+} .
$$

However, as there is no proliferation, oxygen consumption for such a tissue is different. Given that all the ATP produced is used for maintenance $\left(\dot{Q}_{0}+\dot{Q}_{A}=0\right)$, the consumption term is

$$
\mathrm{q}(\phi, c)=-\hat{\theta} \phi
$$

or, in terms of $\alpha$ and $\theta$,

$$
\mathrm{q}(\phi, c)=-\frac{N \alpha \theta}{6} \phi
$$

\section{Boundary and initial conditions}

Initially both tissues are in stress free condition:

$$
\phi(\boldsymbol{x}, 0)=\phi_{0}
$$

There are five boundaries in the model: $\partial \Omega_{\text {west }}, \partial \Omega_{\text {east }}, \partial \Omega_{\text {south }}, \partial \Omega_{\text {north }}$, the four exterior boundaries of model domain $\Omega$, and $\partial \Omega_{t h}$, the boundary between $\Omega_{t}$ and $\Omega_{h}$, or tumour-host interface. 


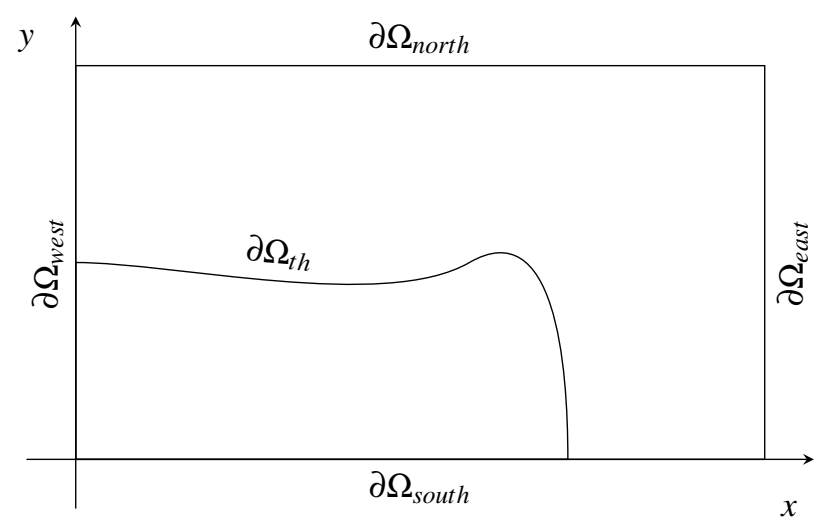

Figure 4: Domain boundaries $\partial \Omega_{\text {west }}, \partial \Omega_{\text {east }}, \partial \Omega_{\text {south }}, \partial \Omega_{\text {north }}$ and tumour-host interface $\partial \Omega_{t h}$.

\section{Domain boundary}

We distinguish three roles of an exterior boundary:

- a boundary coinciding with a blood vessel,

- a remote boundary,

- a symmetry axis.

For boundaries coinciding with a vessel, we assume that there is no penetration of the cells into the vessel, and oxygen supply is always sufficient to maintain its constant concentration $c_{i n}$ :

$$
\frac{\partial(\phi \Sigma(\phi))}{\partial \boldsymbol{n}}(\boldsymbol{x}, t)=0, \quad c(\boldsymbol{x}, t)=c_{i n}, \quad \text { for } \boldsymbol{x} \text { at vascular boundaries }
$$

where $\boldsymbol{n}$ is a unit exterior normal of $\Omega$. These boundaries are the only source of oxygen for the region of tissue in consideration.

For remote boundaries we assume that they stay undisturbed by the growth and there is zero flux of oxygen through them:

$$
\phi(\boldsymbol{x}, t)=\phi_{0}, \quad \frac{\partial c}{\partial \boldsymbol{n}}(\boldsymbol{x}, t)=0, \quad \text { for } \boldsymbol{x} \text { at remote boundaries. }
$$

In most simulations we assume that growth is symmetrical with respect to one of the domain boundaries, and for that boundary

$$
\frac{\partial \phi \Sigma(\phi)}{\partial \boldsymbol{n}}(\boldsymbol{x}, t)=0, \quad \frac{\partial c}{\partial \boldsymbol{n}}(\boldsymbol{x}, t)=0, \quad \text { for } \boldsymbol{x} \text { at a symmetry axis. }
$$




\section{Tumour-host interface}

The tumour-host interface $\partial \Omega_{t h}$ coincides with the material boundary of tumour cells fractions and moves with the velocity of the cells $\boldsymbol{v}$.

We assume continuity of stress on the tumour-host interface $\partial \Omega_{t h}$ :

$$
\lim _{\epsilon \rightarrow+0} \phi \Sigma(\phi)(\boldsymbol{x}+\epsilon \boldsymbol{n}, t)=\lim _{\epsilon \rightarrow+0} \phi \Sigma(\phi)(\boldsymbol{x}-\epsilon \boldsymbol{n}, t) .
$$

If the function $\Sigma(\phi)$ is the same for both tissues then the condition above implies continuity of $\phi$. The kinematic constraint and mass preservation reasons require that the velocity of the interface between the immiscible tissue should satisfy

$$
\boldsymbol{v}_{\partial \Omega_{t h}} \cdot \boldsymbol{n}=-\frac{1}{\Lambda} \nabla(\phi \Sigma(\phi)) \cdot \boldsymbol{n}
$$

\section{Model scaling}

We shall use tildes to mark non-dimensional variables in this Section. In the subsequent Sections we shall use only non-dimensional formulation of the problem and shall omit tildes.

Given that a tissue is a saturated porous medium, and that $\phi+\phi_{l}=\phi_{*}$, one may rescale the volume fraction of cells:

$$
\phi=\tilde{\phi} \phi_{*} .
$$

This way $\tilde{\phi}=1$ would represent the highest possible cell packing density.

The oxygen concentration is scaled with respect to the concentration of oxygen in the blood vessel:

$$
c=\tilde{c} c_{i n} .
$$

Given a spatial scale $L$ and a time scale $\tau$

$$
x=\tilde{x} L, \quad y=\tilde{y} L, \quad t=\tilde{t} \tau,
$$

equations (2.14) and (2.15) may be rewritten as

$$
\frac{\partial \tilde{\phi}}{\partial \tilde{t}}-\frac{\phi_{*} \tau}{L^{2} \Lambda} \widetilde{\operatorname{div}}\left(\tilde{\phi} \widetilde{\nabla}\left(\tilde{\phi} \Sigma\left(\tilde{\phi} \phi_{*}\right)\right)\right)=\frac{\tau \Gamma\left(\tilde{\phi} \phi_{*}, \tilde{c} c_{i n}\right)}{\rho \phi_{*}}
$$

and

$$
\frac{\partial \tilde{c}}{\partial \tilde{t}}=\frac{D \tau}{L^{2}} \tilde{\Delta} \tilde{c}+\frac{\tau}{c_{i n}} \mathrm{q}\left(\tilde{c} c_{i n}, \tilde{\phi} \phi_{*}\right) .
$$

With our choice of the cell growth term (2.17) the corresponding function in non-dimensional variables is

$$
\Gamma(\tilde{\phi}, \tilde{c})=\gamma \phi_{*} \tilde{\phi} \cdot\left(\mathrm{f}\left(\tilde{\phi} \phi_{*}\right) \mathrm{g}\left(\tilde{c} c_{i n}\right)-\theta\right)_{+}-\varepsilon \phi_{*} \tilde{\phi} \cdot\left(\theta-\mathrm{f}\left(\tilde{\phi} \phi_{*}\right) \mathrm{g}\left(\tilde{c} c_{i n}\right)\right)_{+} \cdot
$$


This allows to couple the temporal scale $\tau$ with the rate of tumour proliferation:

$$
\tau=\frac{\rho}{\gamma}
$$

We chose the spatial scale $L$ to be the diffusion distance of oxygen:

$$
L=\sqrt{D \tau}=\sqrt{\frac{D \rho}{\gamma}} .
$$

Thus the equation for the cellular phase is written as

$$
\frac{\partial \tilde{\phi}}{\partial \tilde{t}}-\mu \widetilde{\operatorname{div}}(\tilde{\phi} \widetilde{\nabla}(\tilde{\phi} \widetilde{\Sigma}(\tilde{\phi})))=\widetilde{\Gamma}(\tilde{\phi}, \tilde{c})
$$

where

$$
\mu=\frac{\phi_{*}}{\Lambda D}
$$

is the motility coefficient of the cellular phase, and the functions $\widetilde{\Sigma}$ and $\widetilde{\Gamma}$ have been defined as follows:

$$
\begin{gathered}
\tilde{\Sigma}(\tilde{\phi})=\Sigma\left(\tilde{\phi} \phi_{*}\right), \\
\widetilde{\Gamma}(\tilde{\phi}, \tilde{c})=\tilde{\phi}\left(\mathrm{f}\left(\tilde{\phi} \phi_{*}\right) \mathrm{g}\left(\tilde{c} c_{i n}\right)-\theta\right)_{+}-\tilde{\varepsilon} \tilde{\phi}\left(\theta-\mathrm{f}\left(\tilde{\phi} \phi_{*}\right) \mathrm{g}\left(\tilde{c} c_{i n}\right)\right)_{+},
\end{gathered}
$$

with the rescaled death rate $\tilde{\varepsilon}$

$$
\tilde{\varepsilon}=\frac{\varepsilon}{\gamma} .
$$

Given the nutrient consumption function (2.18), one may rewrite the nutrient equation as

$$
\frac{\partial \tilde{c}}{\partial \tilde{t}}=\widetilde{\Delta} \tilde{c}-\tilde{\alpha} \tilde{\phi} \mathrm{f}\left(\tilde{\phi} \phi_{*}\right) \mathrm{g}\left(\tilde{c} c_{i n}\right)
$$

where $\tilde{\alpha}$ has been rescaled:

$$
\tilde{\alpha}=\frac{\rho \phi_{*}}{\gamma c_{i n}} \alpha
$$

From here on we shall refer to (4.1-4.2) as a non-dimensional model of tissue growth.

\section{Numerical method}

The complete source code of the implemented numerical method is publicly available for download, investigation and use at http://code.google.com/p/cord/. It is distributed under the terms of the GNU General Public License. In this paper we refer to version 0.3 .5 of the programme.

The problem was splitted into a number of more simple equations with respect to physical processes [30]. We splitted the system into 
- system of ODE (proliferation/death of the cells),

- parabolic equation (spatial movement of tumour tissue),

- Poisson equation (oxygen distribution),

- propagation of the boundary with the velocity of tumour cells (tumour geometry changes).

The method for the numerical solution of problem (2.14-3.6) in the non-dimensional form (4.1-4.2) is based on a finite difference approach. Finite difference approximations were constructed on a rectangular grid.

Five-point stencil on a grid $u_{i, j}$ was used to approximate reaction-diffusion equations

$$
\frac{\partial u}{\partial t}=\operatorname{div}(\mathrm{D}(\boldsymbol{x}, u) \nabla u)+\mathrm{R}(\boldsymbol{x}, u)
$$

with second order accuracy in space and first order accuracy in time:

$$
\begin{aligned}
& \frac{u_{i, j}^{n+1}-u_{i, j}^{n}}{\delta t}=\frac{2}{h_{x, i}+h_{x, i-1}}\left(\frac{u_{i+1, j}^{\sigma}-u_{i, j}^{\sigma}}{h_{x, i}} \frac{D_{i+1, j}+D_{i, j}}{2}-\frac{u_{i, j}^{\sigma}-u_{i-1, j}^{\sigma}}{h_{x, i-1}} \frac{D_{i, j}+D_{i-1, j}}{2}\right) \\
& \quad+\frac{2}{h_{y, j}+h_{y, j-1}}\left(\frac{u_{i, j+1}^{\sigma}-u_{i, j}^{\sigma}}{h_{y, j}} \frac{D_{i, j+1}+D_{i, j}}{2}-\frac{u_{i, j}^{\sigma}-u_{i, j-1}^{\sigma}}{h_{y, j-1}} \frac{D_{i, j}+D_{i, j-1}}{2}\right)+R\left(u_{i, j}^{n}\right) .
\end{aligned}
$$

Both implicit $(\sigma=n+1)$ and explicit $(\sigma=n)$ methods are implemented as well as alternating directions implicit method [29]. The reaction term $\mathrm{R}$ and the effective diffusion coefficient $\mathrm{D}$ are always approximated using the values of $u_{i, j}^{n}$ from the previous time step.

Poisson equation is solved by either relaxation to the steady state solution of the reactiondiffusion equation or directly resolving the corresponding system of linear equations.

To track the tumour-host interface $\partial \Omega_{t h}$ we chose level-set method [28], as it works well for proof-of-idea implementation. While this method allows easy capturing of the interface position and topology, it does not conserve the mass and is not suitable for quantitative modelling of tumour tissue.

Given a continuous function $\psi(\boldsymbol{x}), \psi>0$ for tumour tissue and $\psi<0$ for host tissue initially, the location of the tumour-host interface is given by the zero level set of $\psi$. This property of $\psi$ is conserved if the evolution of $\psi$ is described by

$$
\frac{\partial \psi}{\partial t}+\boldsymbol{v} \cdot \nabla \psi=0
$$

where $\boldsymbol{v}=-\mu \nabla(\phi \Sigma(\phi))$ is the cellular phase velocity which coincides with the velocity of the interface at $\partial \Omega_{t h}$. Reinitialization of $\psi$ to the signed distance near the interface is performed by finding the steady state solution $\psi_{0}$ of

$$
\frac{\partial \psi_{0}}{\partial t}=\frac{\psi}{\sqrt{\psi^{2}+w^{2}\left(h_{x}^{2}+h_{y}^{2}\right)}}\left(1-\left|\nabla \psi_{0}\right|\right)
$$


where $w^{2}=16$ is a heuristic parameter affecting the width of the transition region of $\psi_{0}$.

Hybrid methods combining level-set technique and volume of fluid method may be used in future implementations of the numerical method to ensure conservation of mass $[21,24,25]$.

Systems of linear equations are solved using either direct solvers from the UMFPACK library [14, 13, 16, 15] or appropriate iterative solvers [5] (conjugate gradient is used whenever possible). Thomas algorithm is used in ADI method [29]. ODE integration relies on forth order Runge-Kutta method [12].

Blitz++ library [35] has been used for array operations. Hierarchical Data Format 5 [33] was chosen for persistent storage of the state of the model and for representation of results.

We chose to use Cartesian coordinates for simulations. It is a good tradeoff between simplicity of numerical implementation and its ability to simulate both axial-symmetric growth and growth of adjacent cords. We believe it is sufficient for qualitative simulations. However, for reliable quantitative simulations in axial symmetric case we need cylindrical coordinates, and for quantitative simulations of asymmetric case with multiple cords we need 3D implementation.

\section{Simulations}

\section{Growth along a vessel}

The basic configuration of the model is growth with only one blood vessel present. In this configuration the boundary $\partial \Omega_{\text {south }}$ is supposed to concide with the vessel wall, $\partial \Omega_{\text {west }}$ is a symmetry axis, and $\partial \Omega_{\text {east }}, \partial \Omega_{\text {north }}$ are remote boundaries.

We consider a square domain $\Omega=\{x, y: x \in[0,1], y \in[0,1]\}$. Tumour tissue is assumed to occupy initially a region near the vessel $\left\{x, y: \sqrt{x^{2}+y^{2}}<r_{0}\right\}, r_{0}=0.2$.

The following model parameters were used in the simulation: stress free packing density $\phi_{0}=$ $0.75, \Sigma(\phi)=\phi-\phi_{0}$, cell motility $\mu=0.01$, upkeep per cell $\theta=0.15$, death rate $\varepsilon=0.8$, oxygen uptake rate $\alpha=200$, ATP production per cell $\mathrm{f}(\phi)=1-\phi$, oxygen utilization effectiveness $\mathrm{g}(c)=c$.

Initially the growth is almost isotropic until hypoxic region is formed at the periphery of the cord (Figure 5a,b). Later stages of the growth feature almost unidirectional growth along the vessel (Figure 5c,d,e).

The cord reaches and maintains fixed radius, and grows as long as computational domain permits. Outer part of the tumour strip tends to stay in hypoxic conditions and tissue degradation processes prevail there (cell packing density is below stress free value). On opposite, the inner part of the tumour strip, next to the vessel, actively proliferates (Figure 5f).

An important observation is that in the region of the fixed radius, away from the tip of the cord, the distribution of cells is rather plane. The highest packing density of the cells is observed near the very tip of the cord.

The simulation software allows to enable oxygen consumption in the normal host tissue and destruction of the host tissue in hypoxic conditions, as defined by equations (2.19), (2.20). The results are similar to those with no host consumption (Figure 6). The uptake of oxygen in the host 


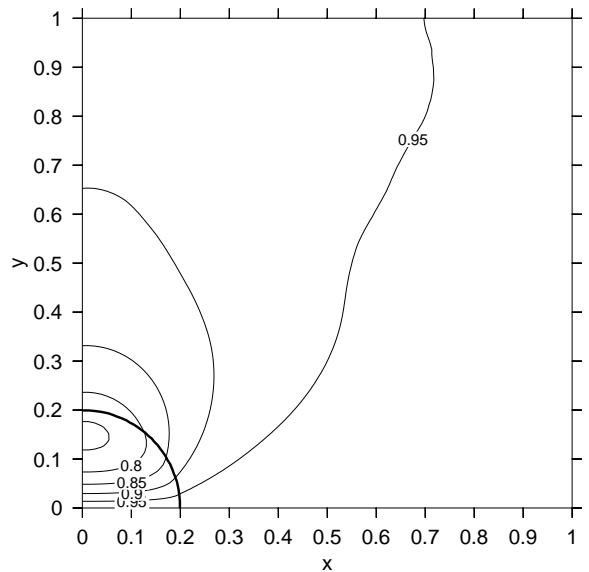

(a) $c, t=0$, initial configuration

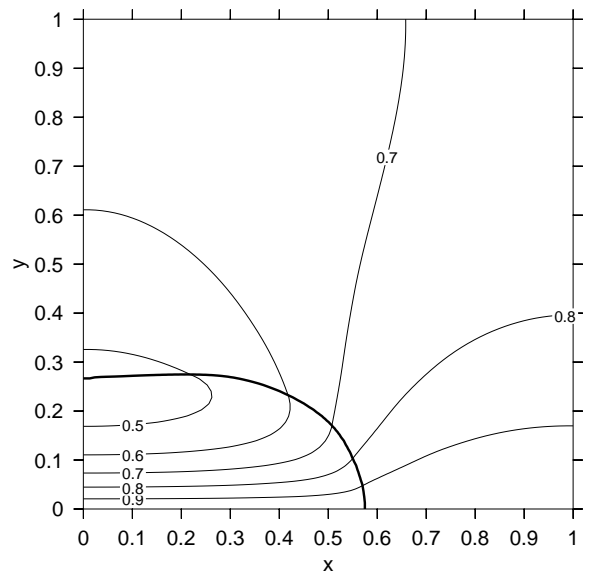

(c) $c, t=400$

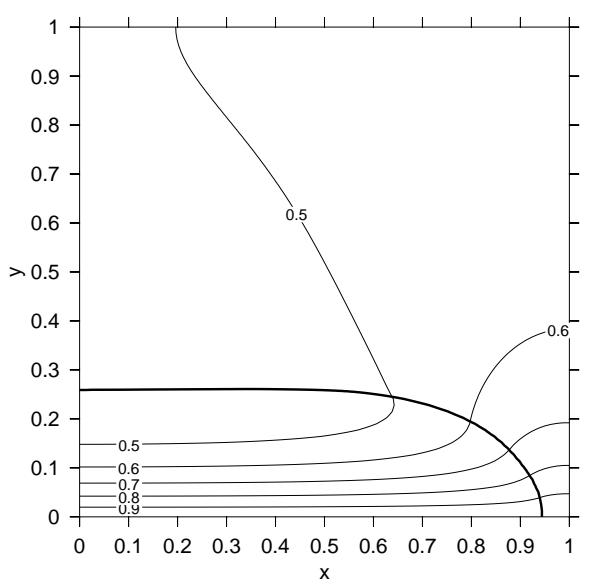

(e) $c, t=700$

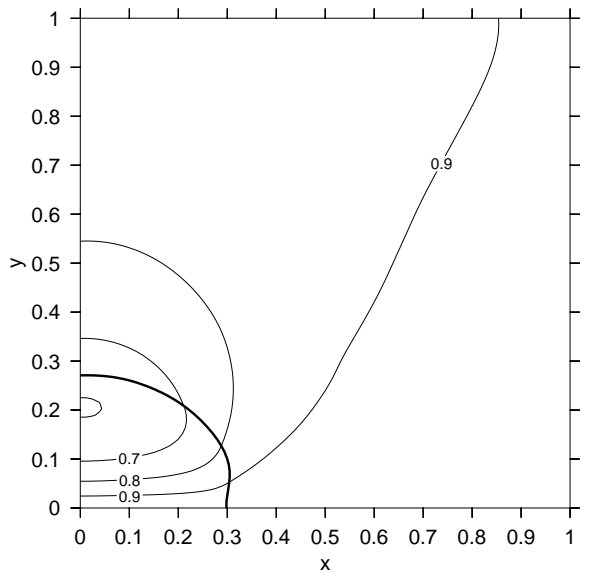

(b) $c, t=100$, end of spherical expansion

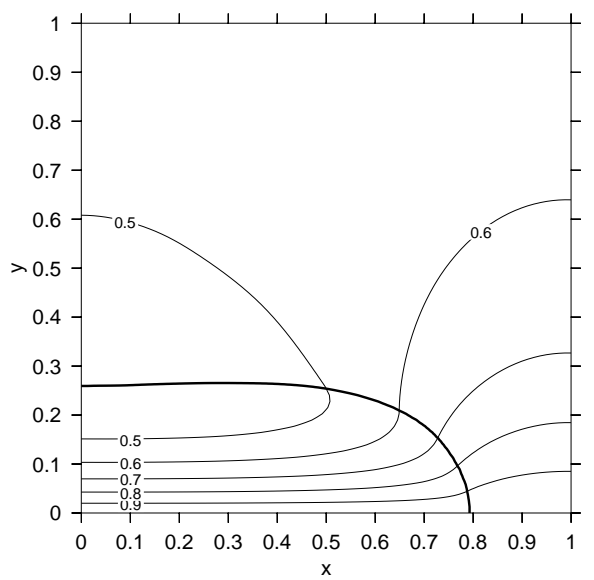

(d) $c, t=600$

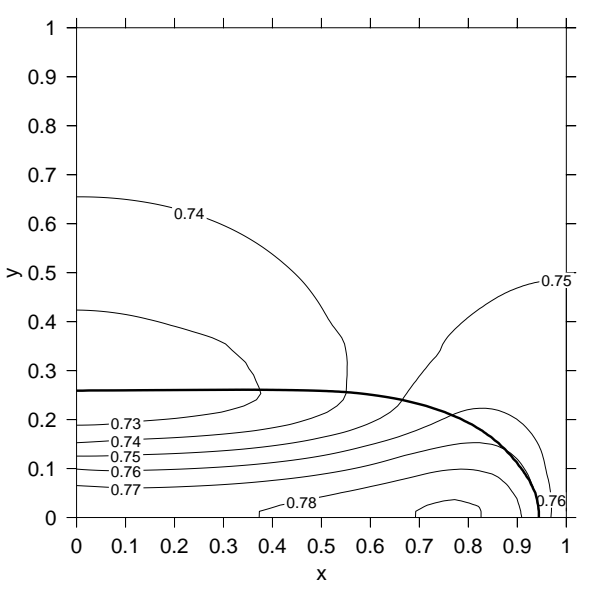

(f) $\phi, t=700$

Figure 5: Cord growth along the vessel. Position of the tumour-host interface is shown by the thick line. (a-e) Isolines show oxygen concentration $c$. (f) Isolines show cell packing density $\phi$. 


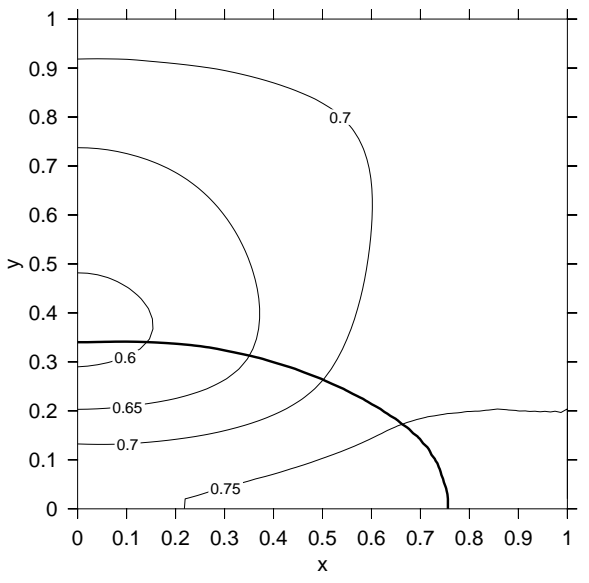

(a) $\phi, t=600$

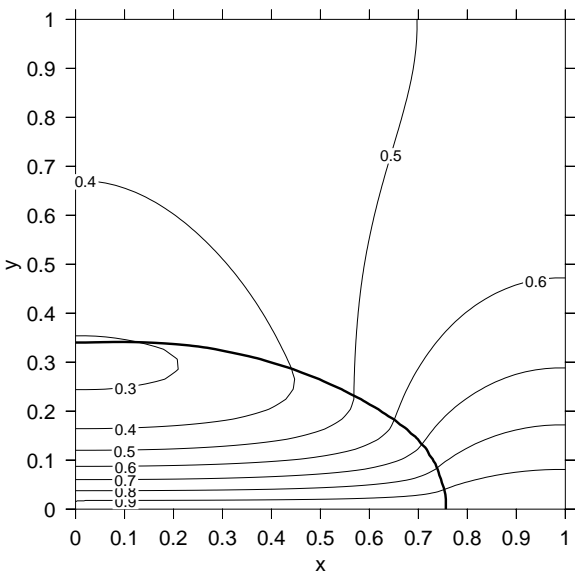

(b) $c, t=600$

Figure 6: Oxygen consumption and cellular death in hypoxic conditions in the outer host tissue lead to the formation of a slightly larger cord with a sharper tip. However, the rate of cord expansion along the vessel is almost the same (compare with Figure 5d).

tissue is lower than that of the tumour, because the former needs just to cover the life maintenance cost, while the latter needs to support the growth. However, the necrosis of the host tissue in hypoxic conditions evidently accounts for the increased width of the cord and its shape alterations.

\section{Growth near the branching point}

Another configuration of interest is a branching point of the vessel. We imitate it with two vessels at $\partial \Omega_{\text {south }}$ and $\partial \Omega_{\text {west }}$. $\partial \Omega_{\text {north }}$ and $\partial \Omega_{\text {east }}$ act like remote boundaries.

Initial condition and parameters are the same as in the previous case, save the higher oxygen uptake rate $\alpha=400$, to balance extra supply of oxygen.

One might observe that there are two sprouts of the cord formed, each of them behaves almost identically to the single cord from the first simulation (Figure 7).

This allows to assume that the modelling approach discussed might be used to describe tumour expansion along the vascular network.

\section{Growth between collateral vessels}

Slightly different results are observed when there are two "parallel" vessels, and initially the tumour has access to only one of them. This simulation aims to show if the tumour spreads to the healthy blood vessels in neighbourhood.

We imitate this situation with $\partial \Omega_{\text {north }}, \partial \Omega_{\text {south }}$ being vascular boundaries, $\partial \Omega_{\text {west }}$ being a symmetry boundary, and $\partial \Omega_{\text {east }}$ being a remote boundary. Starting from the same initial conditions as in the first simulations, and considering the same domain (i.e., the distance between the "vessels" was 1.0), we observed that cord expansion is almost the same as with only one vessel (Figure 8c,d). 


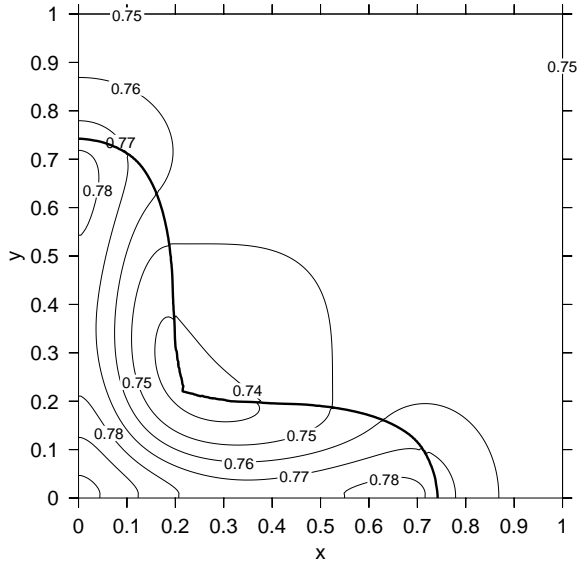

(a) $\phi, t=500$

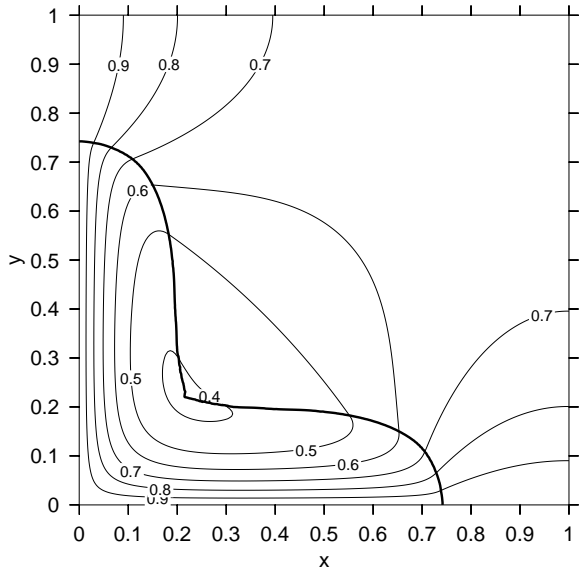

(b) $c, t=500$

Figure 7: Cord growth near the branching point (vessels at $\partial \Omega_{\text {west }}$ and $\partial \Omega_{\text {south }}$ ). Each of the sprouts behaves identically to the single cord.

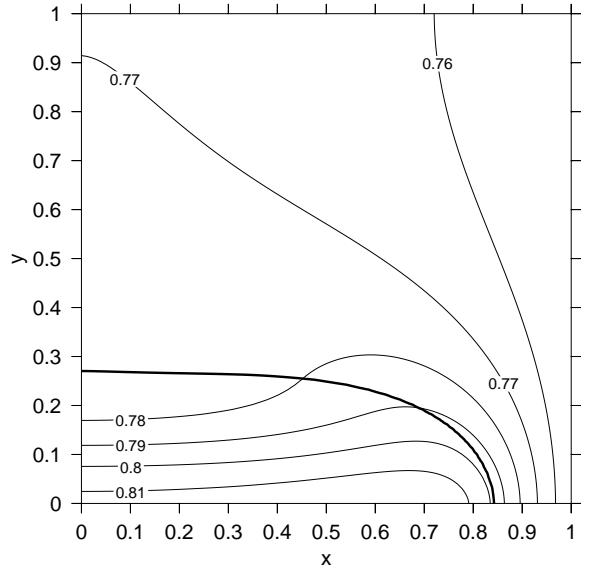

(a) $\phi, t=500, \Omega=[0 ; 1] \times[0 ; 1]$

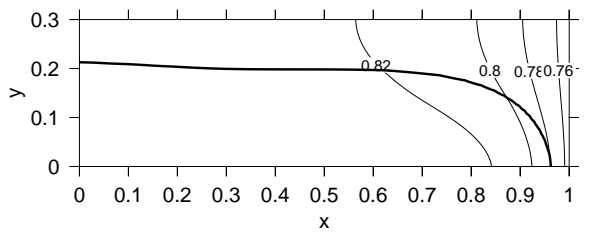

(c) $\phi, t=600, \Omega=[0 ; 1] \times[0 ; 0.3]$

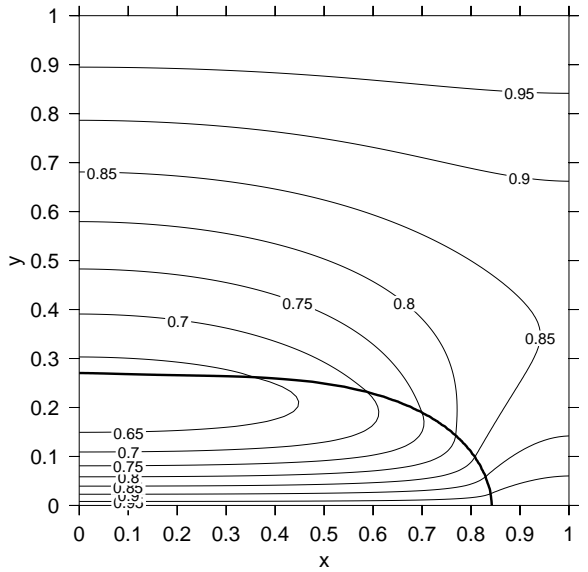

(b) $c, t=500, \Omega=[0 ; 1] \times[0 ; 1]$

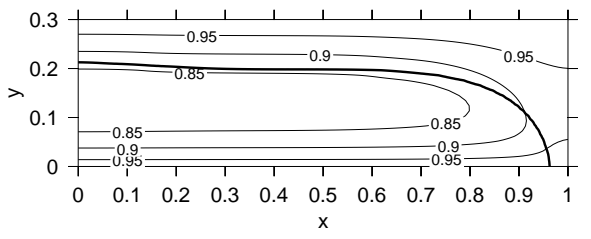

(d) $c, t=600, \Omega=[0 ; 1] \times[0 ; 0.3]$

Figure 8: Cord growth between the collateral vessels. The host tissue outside is compressed and does not permit cooption of the second vessel. 
Basically, the cord does not feel a distant vessel, if it is far enough. Though the expansion of the cord is faster.

However even if we reduce distance between the vessels to the typical width of the cord, the cord still grows along the same vessel that it has been initially put next to. Cooption of the second vessel does not happen (Figure 8c,d).

Next to the second vessel we might observe the host tissue being compressed. It is this tissue that prevents tumour to coopt the second vessels, as tissues remain segregated in our model.

Concentration of oxygen in that region is relatively high. So even if we enable host tissue degradation in prolonged hypoxic condition, that will not help tumour to coopt the second vessel.

Thus we propose that to observe cooption of distant vessels by tumour the model should be extended, to take into account:

- either some kind of tumour toxicity, which destructs the host tissue,

- or ability of cells of different tissues to mix with each other.

Without such mechanisms the model is not able to explain the switch from single cord configuration studied here to the array of parallel cords studied in $[6,7]$.

\section{Effects of tumour-matrix interaction on cord progression}

Let us again consider growth along a vessel, with host tissue inactive. In this case one might notice that the length of the cord (size along $x$-axis) grows almost linearly while the width is almost constant (Figure 9). Thus we may introduce an expansion velocity of the cord as the velocity of its tip.

Having tried the same simulation with various $\mu$, we observed that the expansion velocity strongly

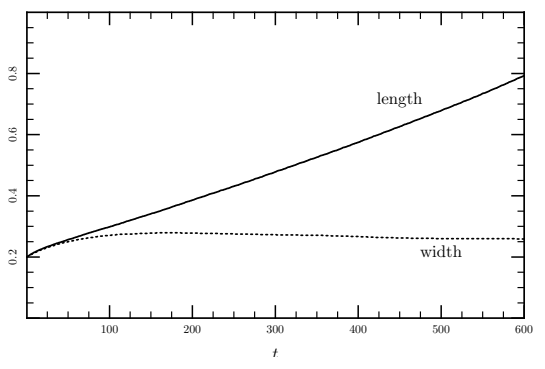

Figure 9: Extents of the cord. Length grows almost linearly. depends on motility of the cellular phase $\mu$ (Figure 10a).

In fact there $\mu$ is inversely proportional to friction forces between the cells and the matrix. The higher is $\mu$, the faster is the relaxation of the tissue. Yet dependency of the velocity on $\mu$ is not monotonic.

For smaller $\mu$ velocity increases as $\mu$ increases, until a certain threshold ( $\mu \approx 0.1$ in our case). Further increase of $\mu$ results in the slow down of the expansion, until it stops almost completely (for $\mu>0.2$ ).

Thus the cord cannot expand if its interaction with the matrix is very weak (high $\mu$ ), yet too strong adhesive forces (low $\mu$ ) may also restrain expansion.

This reasoning is partially confirmed by Figure $10 \mathrm{~b}$, where the maximum of packing density of cells is shown for various $\mu$. We may see that the higher is $\mu$, the lower is $\max (\phi)$, that means that 
a density profile of $\phi$ is smoother, and an average value of $\nabla \phi$ is smaller, thus the force, driving cord expansion is diminished.

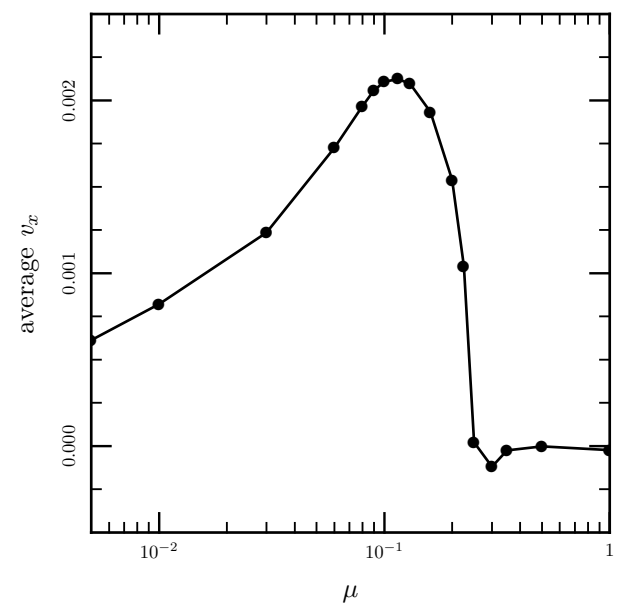

(a)

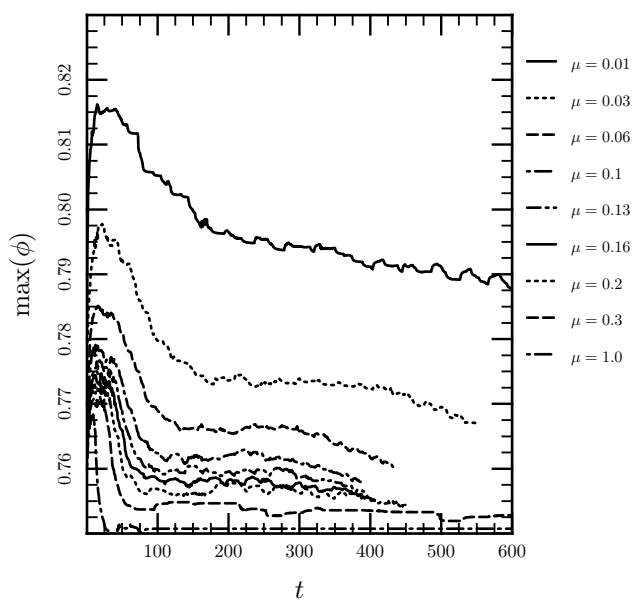

(b)

Figure 10: Effects of tumour-matrix interaction on cord progression. Higher $\mu$ means smaller friction between cells and the matrix and faster relaxation of the tissue. (a) average velocity of tumour expansion along the $x$-axis. (b) maximal packing density of cells for various $\mu$ (simulations were terminated when the cord reached the "remote" boundary).

\section{Conclusion}

Inherent ability of the model to describe the surrounding host tissue, as well as the cord, renders the model into an attractive framework for modelling of tumour-host interactions, transport of drugs and metabolites, and, finally, tumour therapy and evolution.

Two dimensional implementation in cartesian coordinates certainly limits this simulation to qualitative results only. Reliable quantitative results will require complete three dimensional formulation or at least axially symmetric formulation in cylindrical coordinates. More attention should be paid to the implementation of the interface tracking method, especially in the case of more complex geometries. Boundary conditions for oxygen are likely to be reconsidered if angiogenic factors like VEGF become involved in the model. Assumption about identical mechanical properties of tumour and host tissues is another candidate for elimination.

Further extensions of the model might include switch to altered glucose metabolism, tissue acidification, self-sustained growth, anoikis evasion and invasive behaviour.

However, even the simplified model presented here allows to describe proliferative structure of a tumour cord growing in vivo, its expansion, formation of its necrotic shell and its transition to the 
quasi-stationary state. Distance to vessel seems to have decisive influence on the local conditions inside the cord. Dynamics of tumour cord expansion observed in this simulation resembles that of travelling wave, but this needs further investigation.

\section{Acknowledgements}

The research project was partially funded by the Marie Curie Research Training Network MCRTN-CT-2004-503661 "Modelling, Mathematical Methods and Computer Simulation of Tumour Growth and Therapy".

\section{References}

[1] D. Ambrosi, L. Preziosi. On the closure of mass balance models for tumor growth. Mathematical Models and Methods in Applied Science, 12 (2002), No. 5, 737-754.

[2] M. Andreef, D. Goodrich, A. B. Pardee. Cancer Medicine 6 / editors, Donald W. Kufe ... [et al.]., chapter Cell proliferation and differentiation. Hamilton, Ont. ; Lewiston, NY : BC Decker, 2003.

[3] S. Astanin, L. Preziosi. Selected Topics on Cancer Modelling: Genesis - Evolution - Immune Competition - Therapy, chapter Multiphase Models of Tumour Growth, 223-253. Birkhäuser, 2008.

[4] S. Astanin, L. Preziosi, A. Tosin. Modelling tumour cord growth along the source of nutrient. Issledovano v Rossii, (2006). E-publication http: / / zhurnal . ape.relarn.ru/ articles/2006/048.pdf, in Russian.

[5] R. Barrett, M. Berry, T. F. Chan, J. Demmel, J. Donato, J. Dongarra, V. Eijkhout, R. Pozo, C. Romine, H. V. der Vorst. Templates for the Solution of Linear Systems: Building Blocks for Iterative Methods, 2nd Edition. SIAM, Philadelphia, PA, 1994.

[6] A. Bertuzzi, A. Fasano, A. Gandolfi. A free boundary problem with unilateral constraints describing the evolution of a tumor cord under the influence of cell killing agents. SIAM J. Math. Anal., 36 (2004), No. 3, 882-915.

[7] A. Bertuzzi, A. Fasano, A. Gandolfi. A mathematical model for tumor cords incorporating the flow of interstitial fluid. Math. Models Methods Appl. Sci., 15 (2005), No. 11, 1735-1777.

[8] A. Bertuzzi, A. Fasano, A. Gandolfi, D. Marangi. Cell kinetics in tumour cords studied by a model with variable cell cycle length. Math. Biosci., 177-178 (2002), 103-125.

[9] A. Bertuzzi, A. Gandolfi. Cell kinetics in a tumour cord. J. Theor. Biol., 204 (2000), 587-599. 
[10] H. M. Byrne, J. R. King, D. L. S. McElwain, L. Preziosi. A two-phase model of solid tumor growth. Appl. Math. Lett., 16 (2003), 567-573.

[11] M. A. J. Chaplain, L. Graziano, L. Preziosi. Mathematical modelling of the loss of tissue compression responsiveness and its role in solid tumour development. Math. Med. Biol., 23 (2006), 197-229.

[12] B. Cough, ed. GNU Scientific Library Reference Manual - 2nd Edition (Paperback). Network Theory Ltd., 2003.

[13] T. A. Davis. Algorithm 832: UMFPACK, an unsymmetric-pattern multifrontal method. ACM Transactions on Mathematical Software, 30 (2004), No. 2, 196-199.

[14] T. A. Davis. A column pre-ordering strategy for the unsymmetric-pattern multifrontal method. ACM Transactions on Mathematical Software, 30 (2004), No. 2, 165-195.

[15] T. A. Davis, I. S. Duff. An unsymmetric-pattern multifrontal method for sparse LU factorization. SIAM Journal on Matrix Analysis and Applications, 18 (1997), No. 1, 140-158.

[16] T. A. Davis, I. S. Duff. A combined unifrontal/multifrontal method for unsymmetric sparse matrices. ACM Transactions on Mathematical Software, 25 (1999), No. 1, 1-19.

[17] R. A. Gatenby, E. T. Gawlinski, A. F. Gmitro, B. Kaylor, R. J. Gillies. Acid-meditated tumour invasion: a multidisciplinary study. Cancer Res., 66 (2006).

[18] R. J. Gillies, R. A. Gatenby. Hypoxia and adaptive landscapes in the evolution of carcinogenesis. Cancer Metastasis Rev., (2007). E-publication.

[19] L. Graziano, L. Preziosi. Modeling of Biological Materials / editors, F. Mollica ... [etal], chapter Mechanics in tumour growth, 267-328. Birkhäuser, 2007.

[20] G. Hirst, J. Denekamp. Tumour cell proliferation in relation to the vasculature. Cell Tissue Kinet., 12 (1979), 31-42.

[21] A. Loubenets. A New Finite Element Method for Elliptic Interface problems. Ph.D. thesis, Royal Institute of Technology (KTH), Sweden (2006).

[22] A. I. Minchinton, I. F. Tannock. Drug penetration in solid tumours. Nature Reviews Cancer, 6 (2006), 583-592.

[23] J. V. Moore, P. S. Hasleton, C. H. Buckley. Tumour cords in 52 human bronchial and cervical squamous cell carcinomas: Inferences for their cellular kinetics and radiobiology. Br. J. Cancer, 51 (1985), 407-413.

[24] M.Sussman. A second order coupled level-set and volume-of-fluid method for computing growth and collapse of vapor bubbles. J. Comput.Phys., 187 (2003), 110-136. 
[25] M.Sussman, G.E.Puckett. A coupled level set and volume-of-fluid method for computing 3D and axisymmetric incompressible two-phase flows. J. Comput. Phys., 162 (2000), 301-337.

[26] Y. Navalitloha, E. S. Schwartz, E. N. Groothuis, C. V. Allen, R. M. Levy, D. R. Groothuis. Therapeutic implications of tumor interstitial fluid pressure in subcutaneous RG-2 tumors. Neuro Oncol., 8 (2006), No. 3, 227-233.

[27] D. L. Nelson, M. M. Cox. I principi di biochimica di Lehninger. Zanichelli, 2002. Translated by M. Averna, E. Melloni, A. Sdraffa.

[28] S. J. Osher, J. A. Sethian. Fronts propagating with curvature dependent speed: algorithms based on Hamilton-Jacobi formulations. J. Comput. Phys., 79 (1988), 12-49.

[29] D. Peaceman, J. H. H. Rachford. The numerical solution of parabolic and elliptic differential equations. J. Soc. Indust. Appl. Math., 3 (1955), 28-41.

[30] A. Samarskii, P. Vabishchevich. Additive scheme for systems of time-dependent equations of mathematical physics. In Numerical Methods and Applications: 5th International Conference, NMA 2002, Borovets, Bulgaria, August 20-24, 2002, Revised Papers, I. Dimov, I. Lirkov, S. Margenov, Z. Zlatev, eds. Springer, 2003. 48-60.

[31] K. Smallbone, R. A. Gatenby, R. J. Gillies, P. K. Maini, D. J. Gavaghan. Metabolic changes during carcinogenesis: Potential impact on invasiveness. J. Theor. Biol., 244 (2007), 703713.

[32] I. F. Tannock. The relation between cell proliferation and the vascular system in a transplanted mouse mammary tumour. Br. J. Cancer, 22 (1968), 258-273.

[33] The HDF Group. Hierarchical Data Format 5 (2007). http://hdfgroup. com/HDF 5 /.

[34] A. Tosin. Multiphase modeling and qualitative analysis of the problem of the growth of tumor cords. Networks and heterogeneous media, 3 (2008), No. 1, 43-83.

[35] T. L. Veldhuizen, D. Gannon. Active Libraries: Rethinking the roles of compilers and libraries. In Proceedings of the SIAM Workshop on Object Oriented Methods for Interoperable Scientific and Engineering Computing (OO’98). SIAM Press, 1998. 